\title{
Retained Intra-Abdominal Hemostatic Materials: A Rare Cause of Abscess, Intestinal Obstruction, and Intestinal Fistula
}

\author{
Gefei Wang, Jianan Ren, Huajian Ren, Guanwei Li, and Zhiwu Hong
}

\begin{abstract}
Background: A retained intra-abdominal surgical sponge is always followed by foreign body granuloma and serious complications such as perforation, intra-abdominal abscess, intestinal obstruction, and intestinal fistula. Hemostatic materials have become increasingly popular and are used to control intra-abdominal hemorrhage, remaining in the abdominal cavity after surgery. Usually there are no symptoms in patients who have retained hemostatic materials because these materials are bioabsorbable and cause no harm. However, if the body fails to absorb the hemostatic materials completely, the retained materials will cause the same problems as the gossypiboma does.

Case Presentation: We report a 37-year-old male who underwent emergency surgery because of closed abdominal trauma, rectum rupture, and intra-abdominal hemorrhage after an automobile accident. Partial rectectomy with sigmoid stoma was performed, however, the hemostatic materials used during the emergency surgery became calcified and caused damage to the intestine, leading to severe post-operative complications including intra-abdominal abscess, intestinal obstruction, and intestinal fistula. The patient was then transferred to our hospital and cured after the removal of the foreign bodies, resection of the terminal ileum fistula with anastomosis, and colostomy closure.
\end{abstract}

Conclusions: Surgeons need to be cautious when leaving hemostatic materials in the abdominal cavity even if they are bioabsorbable.

Keywords: case report; foreign body; gossypiboma; hemostatic materials

$\mathbf{A}$ RETAINED INTRA-ABDOMINAL foreign body is always a problem followed by foreign body granuloma and serious complications such as perforation, abscess, intestinal obstruction, and intestinal fistula [1]. The most common iatrogenic foreign body is a cotton surgical sponge. Bioabsorbable hemostatic materials have become increasingly popular and are used frequently to control hemorrhage during abdominal surgeries and are left in the abdominal cavity after surgery. If the retained hemostatic materials are not absorbed [2], they may act as foreign bodies and cause the same problems as does the gossypiboma. Here we reported a rare case involving a large amount of hemostatic material used and retained in the abdomen causing severe post-operative complications.

\section{Case Presentation}

A 37-year-old male had an automobile accident on July 23, 2015 that caused closed abdominal trauma, rectum rupture, and intra-abdominal hemorrhage. The patient was sent to the hospital where he underwent emergency surgery. During the operation it was found that the ileal mesentery was partially torn causing bleeding. Thus intra-abdominal gauze packing was applied using two pieces of absorbable hemostatic gauze (GC-507, Gelita Medical B.V., Amsterdam, The Netherlands) and two pieces of absorbable stanching satin (Beijing Textile Research Institute, Beijing, China). One box of blend microporous polysaccharide hemospheres (Shandong Success Pharmaceutical Technology Co., Ltd., Jinan, China) was

Department of Surgery, Jinling Hospital, Medical School of Nanjing University, Nanjing, P.R. China.

(C) Gefei Wang et al. 2017; Published by Mary Ann Liebert, Inc. This Open Access article is distributed under the terms of the Creative Commons License (http://creativecommons.org/licenses/by/4.0), which permits unrestricted use, distribution, and reproduction in any medium, provided the original work is properly credited. 
also used to stop the bleeding. The traumatic rupture of the upper rectum was identified, and partial rectectomy with sigmoid stoma was performed. The patient suffered from intermittent fever after the surgery, with the maximum body temperature reaching $39.5^{\circ} \mathrm{C}$. The source of intra-abdominal infection was undetermined and antibiotic treatments demonstrated poor efficacy. The fever persisted for approximately two months and symptoms of incomplete intestinal obstruction appeared repeatedly, which were relieved by conservative treatments.

This patient was transferred to our hospital on December 15, 2015 with the chief complaints of abdominal distension and pain, fever, and sigmoid stoma stopping exhaust and defecation. The abdominal computed tomography (CT) scan showed intra-peritoneal gas and effusion indicating the formation of intra-abdominal abscess. In addition, highdensity shadows of foreign bodies were seen in the mesentery of the terminal ileum. The protrusions at the edges of the foreign bodies were sharp and had pierced the intestinal wall (Fig. 1).

With the aid of bedside ultrasonography to evaluate the location and depth of the intra-abdominal abscess, an experienced surgeon performed percutaneous drainage using a sump drain, a modified closed double-lumen irrigationsuction tube, via trocar puncture. This was an innovative technique to drain the abscess efficiently and has been described in our previous studies [3-5]. Fistulography monitoring the dynamic flow of contrast medium injected from external opening under the radiograph indicated the pres- ence of the fistula of the terminal ileum (Fig. 2). The intraabdominal infection resolved after effective drainage was achieved, and the symptoms of ileus were also relieved. The patient gradually resumed the administration of enteral nutrition. Abdominal CT re-examination confirmed that the intra-abdominal abscess had been controlled completely, however, the high-density shadows of foreign bodies persisted in the mesentery (Fig. 3). The presence of the foreign bodies prevented the spontaneous closure of the fistula of the terminal ileum, and occasionally resulted in the symptoms of incomplete intestinal obstruction. The patient received intra-peritoneal adhesiolysis, resection of the fistula of the terminal ileum with anastomosis, and the colostomy closure on July 5, 2016. Intra-operative findings included the identification of a fistula in the terminal ileum $60 \mathrm{~cm}$ away from the ileocecus, and a hard bone-like substance wrapped in the mesentery of the terminal ileum with sharp protrusions piecing into the intestinal wall and the mesentery. The size of the foreign bodies was approximately $6 \mathrm{~cm} \times 5 \mathrm{~cm} \times 3 \mathrm{~cm}$, and the shape was irregular, resembling corals with sharp spines (Fig. 4). Histopathologic examination of the foreign bodies showed the calcified tissues. The patient recovered after the surgery and was discharged.

\section{Discussion}

A retained foreign body in the abdominal cavity after surgery is always an issue that needs to be addressed. The most common iatrogenic foreign body is cotton surgical
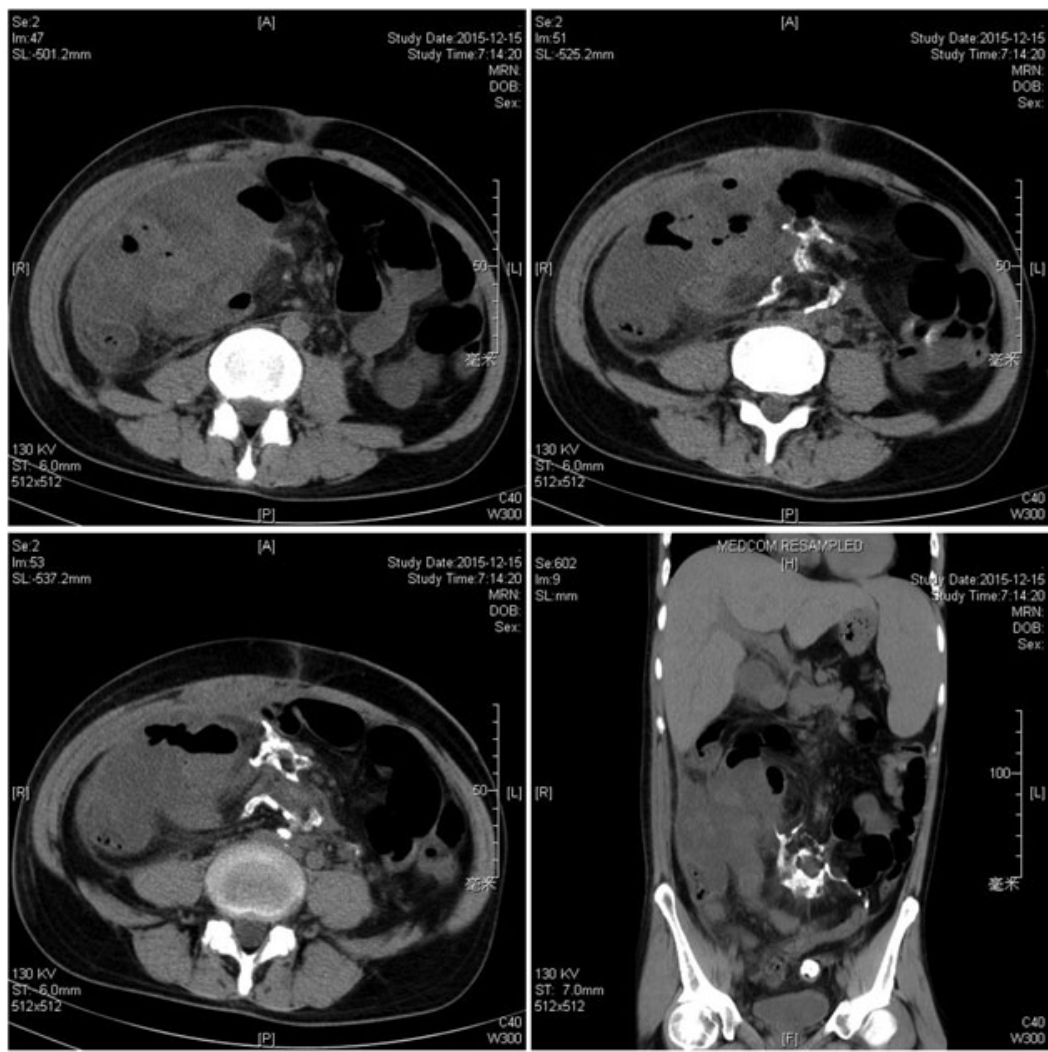

FIG. 1. The abdominal computed tomography (CT) scan showed intra-peritoneal gas and effusion indicating the formation of intra-abdominal abscess. The foreign bodies on CT manifested as high-density shadow seen in the site of the ileal mesentery, of which the protrusions at the edge were sharp and pierced the intestinal wall. 

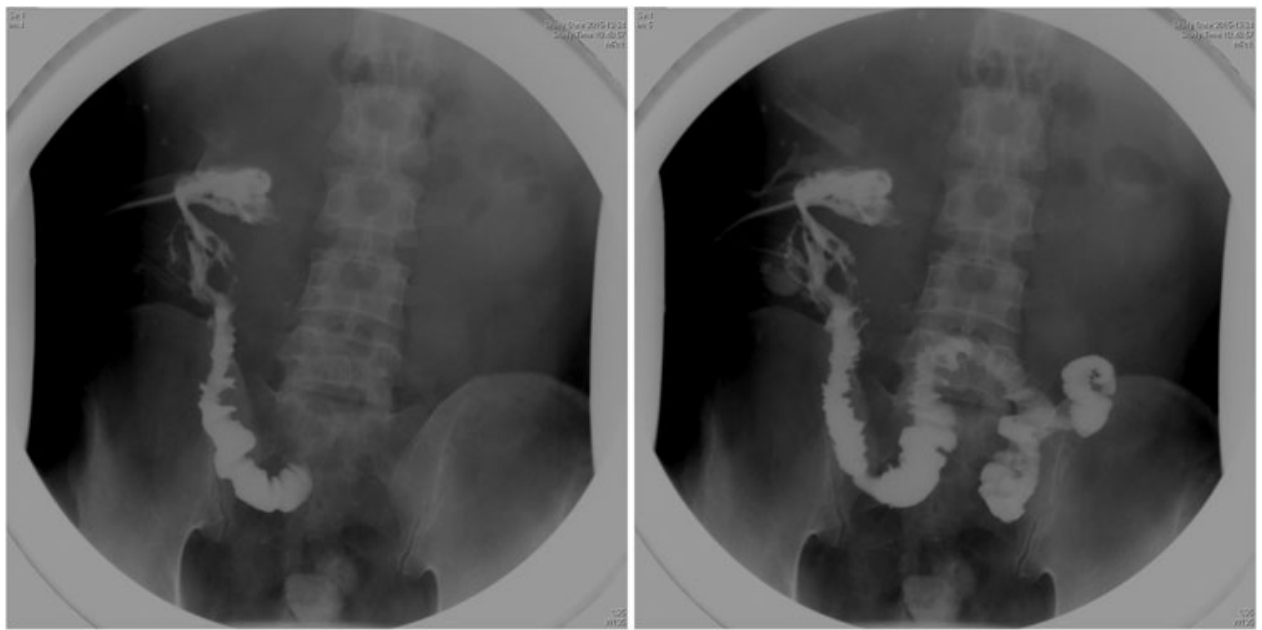

FIG. 2. The contrast medium was injected from the drainage tube and monitored under the radiogrph. The fistulography confirmed the presence of the fistula of the terminal ileum.

sponge, which is called a gossypiboma [6]. The incidence of retained surgical sponge is hard to estimate accurately because of the undiscovered asymptomatic cases and non- reporting of occurrences. The reported incidence is one per 1,000-15,000 abdominal operations. Pain/irritation (42\%), palpable mass $(27 \%)$, and fever (12\%) were the leading signs and symptoms but $6 \%$ of cases were asymptomatic [7]. Gossypiboma can present with foreign body granuloma, even as malignant fibrous histiocytoma [8], and also can cause serious complications such as perforation, intra-abdominal abscess, intestinal obstruction, and intestinal fistula [9,10].

Treatment of gossypiboma relies on the surgical removal of the foreign bodies and management of the damaged viscera, especially the intestine, usually through the previous abdominal incision. Sometimes endoscopic or laparoscopic approaches can be attempted in patients without severe abdominal adhesions. The most common methods to detect the intra-abdominal foreign bodies are CT $(61 \%)$,
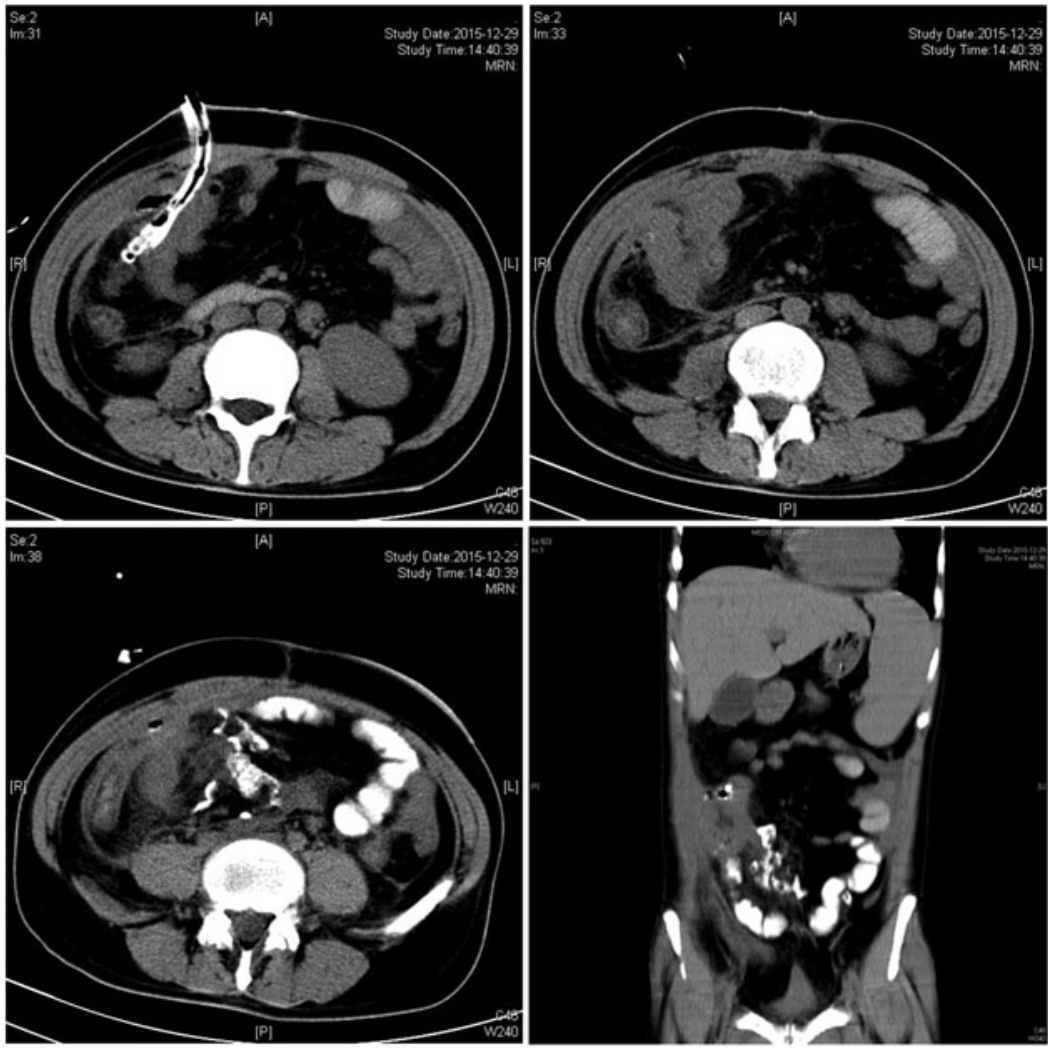

FIG. 3. Abdominal computed tomography (CT) re-examination showed that the intra-abdominal abscess resolved after effective drainage by the sump drain, however, the high-density shadow of foreign bodies still existed. 


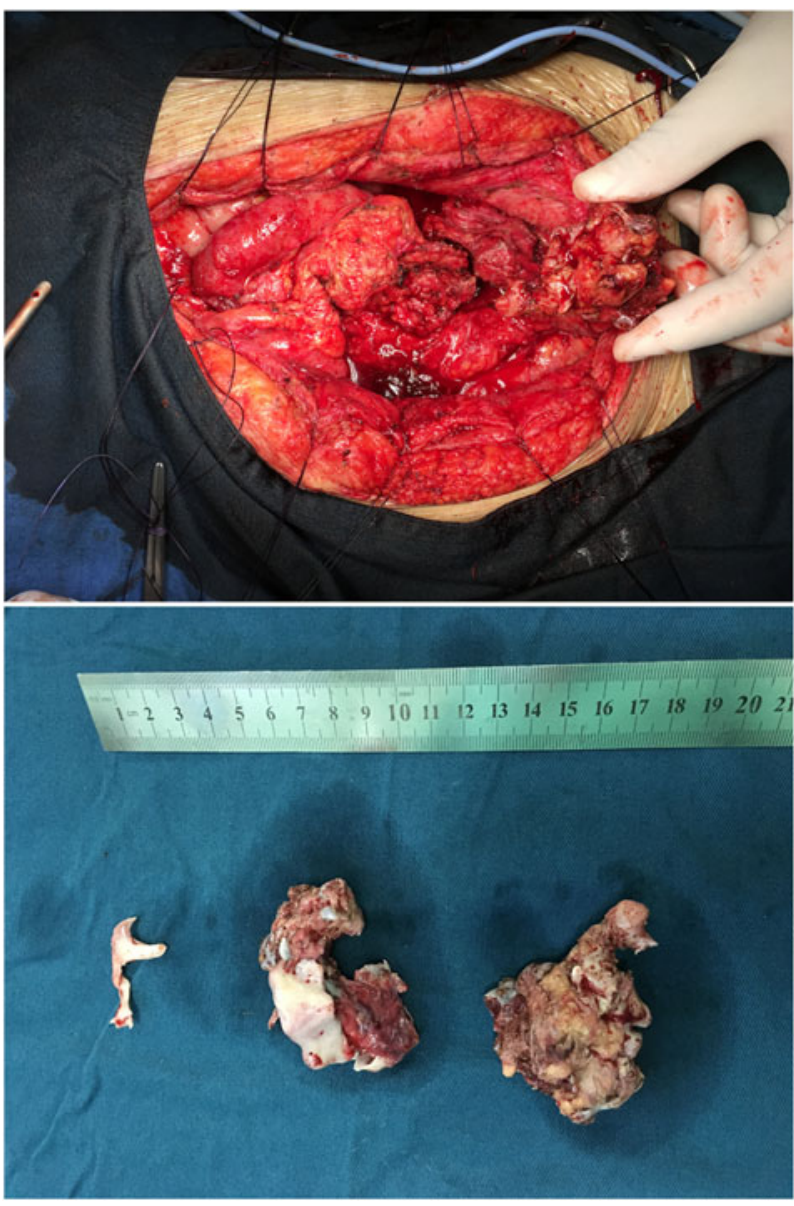

FIG. 4. Intra-operative exploration found a hard and sharp-edged substance wrapped in the ileal mesentery with some of the protrusions piecing the intestinal wall. The foreign bodies had an irregular and coral-like shape, and the granulation tissue had grown into sharp spines.

plain radiography (35\%), and ultrasound (34\%). Thus, the preferred diagnostic modality in the suspected cases should be an abdominal CT scan [11].

In the present practice, hemostatic materials are widely used in abdominal surgery to help stop bleeding of capillaries, veins, and small arteries. Hemostatic materials are considered bioabsorbable, and are often left in the surgical hemorrhagic site. However, the biodegradability depends on the amount used, the implanted site, and environmental factors [12]. If hemostatic materials are not absorbed completely by the body, retained hemostatic materials can act as foreign bodies just like gossypiboma, and may appear as recurrent tumor after surgical resection of the tumor [13] or even cause severe post-operative complications such as intra-abdominal abscess, intestinal obstruction, and intestinal fistula.

Our study reviewed the case of a 37-year-old male patient who underwent emergency surgery to manage the traumatic hemorrhage of mesenteric vessel and rectal rupture caused by an automobile accident. A large amount of hemostatic materials including absorbable hemostatic gauze, stanching satin, and blend microporous polysaccharide hemospheres were used to control the hemorrhage of mesenteric vessels and were left in the abdomen after surgery. Severe compli- cations such as intra-abdominal abscess, intestinal obstruction, and intestinal fistula developed post-operatively because of the poor absorption and calcification of those retained hemostatic materials.

We used a sump drain via trocar puncture for the treatment of the intra-abdominal abscess. This technique has been reported to play a vital role in the source control of intraabdominal infection with great effects [3-5]. However, in this case the symptoms of intestinal obstruction persisted and closure of intestinal fistula could not be achieved by conservative treatments because of the retained hemostatic materials. The definitive surgery was difficult considering severe abdominal adhesions but necessary to remove the retained calcified foreign bodies and to resect the damaged and fistulous part of the ileum.

\section{Conclusion}

In summary, we reported that a surgical patient suffered post-operative intra-abdominal abscess, intestinal obstruction, and intestinal fistula resulting from the retained intra-abdominal hemostatic materials. This was rare but definitely a clinical dilemma of treatment because of the accompanying complications and long courses of disease. In conclusion, surgeons should be cautious leaving hemostatic materials in the abdominal cavity even if these materials are bioabsorbable.

\section{Acknowledgments}

This study was supported by grants from National Natural Science Foundation of China (81571881). Written and signed consent to publish the information was obtained from the patient.

\section{Author Disclosure Statement}

No competing financial interests exist.

\section{References}

1. Sozutek A, Colak T, Reyhan E, et al. Intra-abdominal gossypiboma revisited: Various clinical presentations and treatments of this potential complication. Indian J Surg 2015;77(Suppl 3):1295-1300.

2. Gabay M. Absorbable hemostatic agents. Am J Health Syst Pharm 2006;63:1244-1253.

3. Liu S, Ren J, Gu G, et al. Drainage of intra-abdominal abscesses with gastrointestinal fistula in Crohn's disease using a sump drain via trocar puncture: A modified strategy. Tech Coloproctol 2013;17:459-461.

4. Gu G, Ren J, Liu S, et al. Comparative evaluation of sump drainage by trocar puncture, percutaneous catheter drainage versus operative drainage in the treatment of Intra-abdominal abscesses: A retrospective controlled study. BMC Surg 2015;15:59.

5. Gu G, Ren J, Yuan Y, et al. An innovative technique for intra-abdominal abscess drainage using a sump drain by trocar puncture. Am Surg 2011;77:E166-167.

6. Bani-Hani KE, Gharaibeh KA, Yaghan RJ. Retained surgical sponges (gossypiboma). Asian J Surg 2005;28:109115 .

7. Malhotra MK. Migratory surgical gossypiboma-cause of iatrogenic perforation: Case report with review of literature. Niger J Surg 2012;18:27-29. 
8. Kaplan M, Iyikosker HI. A new complication of retained surgical gauze: Development of malignant fibrous histiocytomaReport of a case with a literature review. World J Surg Oncol 2012; 10:139.

9. Popoola AA, Bello JO, Ezeoke GG, et al. Concealed enterovesical fistula associated with forgotten intra-abdominal haemostat and intravesical towel. Case Rep Urol 2014; 2014:723592.

10. Massimiliano PA, Massimo PS. Retained intra-abdominal surgical instrument: A rare condition of acute abdomen. ANZ J Surg 2010;80:758.

11. Fields RC, DeMatteo RP. Incidentally discovered, intraabdominal metallic foreign object on preoperative imaging. Am Surg 2010;76:E180-181.

12. Tomizawa Y. Clinical benefits and risk analysis of topical hemostats: A review. J Artif Organs 2005;8:137142.

13. Wang $H$, Chen $P$. Surgicel(R) (oxidized regenerated cellulose) granuloma mimicking local recurrent gastrointestinal stromal tumor: A case report. Oncol Lett 2013;5: 1497-1500.
Address correspondence to:

Dr. Jianan Ren

Department of General Surgery

Jinling Hospital

305 East Zhongshan Road

Nanjing, 210002

P.R. China

E-mail: JiananR@gmail.com

\section{Abbreviation Used \\ $\mathrm{CT}=$ computed tomography}

Cite this article as: Wang G, Ren J, Ren H, Li G, Hong Z (2017) Retained intra-abdominal hemostatic materials, a rare cause of abscess, intestinal obstruction, and intestinal fistula. Surgical Infections Case Reports 2:1, 35-39, DOI: $10.1089 /$ crsi.2017.0006 